\title{
Emission of cosmic rays from Jupiter: magnetospheres as possible sources of cosmic rays
}

\author{
G. Pizzella ${ }^{\mathrm{a}}$ \\ Istituto Nazionale di Fisica Nucleare, Laboratori Nazionali di Frascati, Frascati, Italy
}

Received: 28 June 2018 / Accepted: 12 October 2018 / Published online: 22 October 2018

(C) The Author(s) 2018

\begin{abstract}
Measurements made recently with the PAMELA satellite during a period of 8 years have shown with good evidence that a fraction of the cosmic rays detected on Earth comes from Jupiter. This result draws attention to the idea that magnetospheres of astrophysical objects could contribute to the sources of cosmic rays. In this paper we extend our analysis of the cosmic rays measurements made with PAMELA finding further evidence that the cosmic ray fluxes are larger on average when the Earth orbit intersects the lines of the measured interplanetary magnetic field connecting Jupiter with Earth. This effect shows up with more than ten standard deviations, difficult to explain without the idea that part of the cosmic ray protons comes directly from the Jupiter magnetosphere.
\end{abstract}

\section{Introduction}

A recently published paper [1] has provided evidence that a small but not negligible fraction of cosmic rays detected by the Earth satellite PAMELA ${ }^{1}$ comes from the planet Jupiter. The suggestion was that higher energy particles leak out from the Jupiter trapped particle region and then are ejected into space following the interplanetary magnetic field (IMF) lines of force, eventually reaching Earth.

This result draws attention to the idea put forward long ago [3] that magnetospheres of astrophysical objects could contribute to the sources of cosmic rays.

On the other hand it is believed today that pulsars be one of the candidates of the observed ultra-high-energy cosmic rays, getting power by a centrifugal mechanism of acceleration.

\footnotetext{
${ }^{1}$ PAMELA, launched on 15th June 2006, in a 350-600 km heigh orbit, is a space-based experiment designed for precise measurements of charged cosmic rays - protons, electrons, their antiparticles and light nuclei in the kinetic energy interval from several tens of $\mathrm{MeV}$ up to several hundreds of $\mathrm{GeV}$ [2].
}

a e-mail: guido.pizzella@lnf.infn.it
The result obtained with the cosmic rays coming from the Jupiter magnetosphere reinforces the possibility that astrophysical magnetosphere be indeed the source of the cosmic radiation.

In this paper we wish to investigate more thoroughly the experimental result obtained with PAMELA. The use of 8 years of data allows to obtain useful information on statistical basis. The aim is to increase our confidence that astrophysical magnetospheres be a source of cosmic rays.

\section{Trapped radiation in magnetosphere}

An unexpected discovery was the Van Allen belt in 1958 . Although in earlier times Kristian Birkeland, Carl Störmer, and Nicholas Christofilos considered the possibility that fluxes of charged particles could dwell in the magnetic field surrounding the Earth, nobody was expecting so large density of charged particle population that formed a sort of permanent belt surrounding the Earth.

Afterwards many experiments by means of several spacecraft (Pioneer 10 and 11, Voyager 1 and 2, Ulysses. Galileo, Cassini) have shown that all major planets in the solar system possessing a magnetic field (and hence having a magnetosphere) also have Van Allen belts surrounding them [4], in particular Jupiter which has a dipole magnetic field about two thousand times greater than that of the Earth and a trapping region containing very high energy particles.

The natural question is how the Van Allen belts are originated. In the Earth's magnetosphere electromagnetic waves have been detected since the beginning (see [5-7]), presumably originated by the action of the solar wind on the magnetosphere [8] and this justifies the presence of acceleration mechanisms which act on the magnetospheric particles.

Reeves et al. [9] using data from the Van Allen Radiation Belt Storm Probes, launched by NASA on 30 August 2012, discovered that radiation belt electrons are accelerated locally 
by wave-particle interactions. This local energy comes from electromagnetic waves coursing through the belts, tapping energy from other particles residing in the same region of space.

In the Jovian magnetosphere, trapped particles are about ten times more energetic than the ones from the equivalent radiation belts of Earth. In addition, they are several orders of magnitude more abundant [10]. Indication of accelerating mechanisms have also being published [11-13].

In this paper we want to study whether magnetospheric systems, home of accelerating mechanisms, contribute to the cosmic radiation that pervades the Universe.

\section{Generation of cosmic rays in magnetospheres}

Gold [14] suggested that cosmic rays be generated by the explosion of supernovae, but it is likely that there can be a variety of sources like, i.e. active galactic nuclei and, as suggested by Fermi [15], sources of continuos nature acting in the intergalactic space.

The observation [16] of ultrahigh-energy cosmic rays (UHECR), with energies above $6 \times 10^{19} \mathrm{eV}$, obtained with the Pierre Auger Observatory during 3.7 years, suggests that AGN or similar objects could be possible sources.

In the following we discuss, using the cosmic ray detected by PAMELA and the vicinity of the Jovian powerful magnetosphere, whether the energetic protons generated in magnetospheres could contribute to the measured cosmic rays.

We discuss briefly what is the possible largest energy of protons accelerated in a magnetosphere. The protons leak out from the trapping region when their motion violate the adiabatic invariance. This occurs when the Alfven condition (see also the Hillas criterion [17])

$$
\frac{(d B / d r) R_{L}}{B} \leq \epsilon,
$$

is not satisfied anymore $[18,19]$ (B is the magnetic field, $R_{L}$ the Larmor radius and $\epsilon$-the Alfven discriminant- is a dimensionless quantity order of a few percent (in Ref. [3] $\epsilon \sim 5 \%)$.

A prediction of this model is a cosmic cutoff energy given by

$E_{\max }=\frac{\epsilon c q M}{3 r_{S}^{2}}$,

where $r_{S}$ is the radius of the source and $\mathrm{M}$ its magnetic dipole.

In the Van Allen belt of Earth with $M \equiv M_{E}=8 \times$ $10^{15} \mathrm{Tm}^{3}$, the largest energy of protons is of the order of a several $100 \mathrm{MeV}$ [2].

Although the largest energy might depend on other parameters, in addition to those appearing in Eq. 2, for Jupiter, with
$r_{S} \sim 71,500 \mathrm{~km}$ and $M_{J} \sim 1800 \times M_{E}$, we try to scale up from the Earth $500 \mathrm{MeV}$, obtaining

$E_{\max } \sim 70 \mathrm{GeV}$.

This is a very upper limit for Jupiter, greater than the observed proton energy in the Jovian magnetosphere. Because of the complexity of the Jovian magnetosphere (including the effect of its Io satellite) with respect to the Earth's one, it is likely that the real upper limit be smaller.

In the case of cosmic rays of extra solar-system origin, for a neutron star with $r_{S} \sim 10 \mathrm{~km}$ and $M_{N S}=10^{21} \mathrm{Tm}^{3}$, we calculate

$E_{\max } \sim 3 \times 10^{19} \mathrm{eV}$

This value compares with the highest cosmic ray proton energy detected so far. Higher values can be obtained for larger values of $\mathrm{M}$.

\section{Experimental evidence about the Jovian contribution to cosmic rays}

Earth based instrumentation has been considered for studying whether was possible that an even small fraction of the cosmic rays detected by this instrumentation came from Jupiter.

The first attempt was made with the available neutron monitors, operating with rigidity thresholds of the order of $10 \mathrm{GV}$. It was found [20] that the measured cosmic ray flux was enhanced at certain times when considered in a reference system co-rotating with Jupiter. The enhancement was of the order of $0.4 \%$.

Defining the angle $\Phi_{E J}=\overbrace{\text { Jupiter.Sun.Earth }}$, the angle between Jupiter, the Sun and the Earth, the enhancement occurred for values $\Phi_{E J} \sim 60^{\circ}-180^{\circ}$ with maximum at $\Phi_{E J} \sim 125^{\circ}$.

A similar study was made by Mitra et al. [21,22]. They found a strong modulation at the synodic Jupiter period above $99 \%$ confidence level and an angle $\Phi_{E J}=140^{\circ}-160^{\circ}$, and concluded that there was a strong possibility that a part of cosmic rays comes from Jupiter.

A negative result was instead found in [23,24]. They were focused in particular on the idea that the magnetic field of Jupiter could shield the cosmic rays coming from the outer space. Their conclusion was that the Jovian effect on cosmic rays observable at the Earth's ground is minimal.

The indications obtained with the Earth based instrumentation remain rather vague, but an important test can be made with the measurements made during 8 years with the PAMELA satellite.

If charged particles are accelerated in the Jovian magnetosphere and then escape, they should follow the interplanetary 
magnetic field lines, ${ }^{2}$ finally reaching Earth where they are detected within the cosmic rays fluxes.

In the following we start by considering the Interplanetary Magnetic Field (IMF) as measured by interplanetary spacecrafts and then we study the cosmic rays measured on Earth aboard the PAMELA satellite.

\subsection{The interplanetary magnetic field}

The IMF plays an important role, because its lines of force guide the protons with energy up to a few $\mathrm{GeV}$ from their possible source, Jupiter, to Earth.

In the Parker model [25] the IMF originates in the Sun and is transported by the solar wind. If the Sun did not rotate, the magnetic lines would have been radial straight lined. Due to the Sun rotation ( $\Omega \sim 25$ days) the lines become spirals around the Sun [25].

In this model the components of the magnetic field in the ecliptic plane, $B_{r}$ and $B_{t}$, are associated to the two invariants: $B_{r} r^{2}=$ first invariant and $B_{t} r V_{R}=$ second invariant, where $r$ is the distance from the Sun and $V_{R}$ the radial velocity of the solar wind. The first invariant is due to the conservation of the magnetic flux, the second one to the vanishing of the electric field because of the high conductivity of the solar wind plasma.

The equation of the magnetic field lines in the ecliptic plane can be derived from the equation

$\frac{r d \phi}{d r} \simeq \frac{B_{t}}{B_{r}} \frac{V_{R}}{\Omega}=0.9 \frac{V_{R}}{400 \mathrm{~km} / \mathrm{s}} \frac{B_{t}}{B_{r}}$,

where we have put $\Omega=25$ days, the distances are expressed in $\mathrm{AU}$ and the angles in radians. The magnetic lines of force obtained by integrating this equation have the shape of spirals.

It is well known that the IMF, being affected by great turbulence due to the solar activity, has large fluctuations. In this paper we consider 8 years of data which could average out many fluctuations.

We could simply adopt the IMF as given by the Parker theory, but, in my opinion, it is more realistic to consider the measurements of the IMF made with various spacecrafts (Helios 2, IMP8, Pioneer, Venus Orbiter, Voyager 1, Ulysses).

The experiments with space borne magnetic field detectors all give an IMF with spiral lines of force, but the precise behavior depends on the radial gradients of the ratio $B_{t} / B_{r}$, that, in the Parker theory, scales linearly with $r$. Measurements made, in particular, with the Pioneer 11, Voyager and Ulysses spacecrafts [26-32] show some discrepancies from the behavior predicted in the Parker theory.

\footnotetext{
2 This is possible, because $1 \mathrm{GeV}$ protons have a Larmor radius, for a IMF between Jupiter and Earth of about $1 \mathrm{nT}$, of the order of $0.02 \mathrm{AU}$.
}

Table 1 The angle $\Phi_{E J}$ (degree) for $V_{R}=400 \mathrm{~km} / \mathrm{s}$ and $V_{R}=$ $500 \mathrm{~km} / \mathrm{s}$

\begin{tabular}{llll}
\hline$\gamma$ & $\Phi_{E J}$ & $\Phi_{E J}$ & Reference \\
& $V_{R}=400 \mathrm{~km} / \mathrm{s}$ & $V_{R}=500 \mathrm{~km} / \mathrm{s}$ & \\
\hline 1 & 216 & 270 & Parker model [25] \\
0.57 & 140 & 176 & Khabarova [27,32] \\
0.37 & 117 & 146 & Behannon [28] \\
\hline
\end{tabular}

Let us calculate the angle $\Phi_{E J}$, the angle between Jupiter, the Sun and the point where the IMF line intersect the Earth orbit. Integrating Eq. 5 and putting, as already suggested in [28], $B_{t} / B_{r} \sim r^{\gamma}$ we find the magnetic line of force connecting Jupiter with Earth

$\Phi_{E J}=\frac{0.9}{\gamma}\left(r_{\text {Jupiter }}^{\gamma}-r_{\text {Earth }}^{\gamma}\right) \frac{V_{R}}{400 \mathrm{~km} / \mathrm{s}}$,

where $\gamma=1$ in the Parker theory $\left(r_{\text {Jupiter }}=5.2\right.$ and $r_{\text {Earth }}=1$ ) and the angles are expressed in radians.

We show in the Table 1 the angle $\Phi_{E J}$ for the Parker model and that obtained from the measurements made with the space probes, considering that the solar wind velocity varies, roughly, between $V_{R}=400 \mathrm{~km} / \mathrm{s}$ and $V_{R}=500$ $\mathrm{km} / \mathrm{s}$ with occasional peaks up to $V_{R}=700 \mathrm{~km} / \mathrm{s}$.

We notice that the angles $\Phi_{E J}$ where the IMF lines intersect the Earth orbit are smaller than $180^{\circ}$ for a large range of solar wind velocities.

A schematic view of Sun, Earth and Jupiter with the IMF lines connecting Jupiter with Earth is shown in Fig. 1.

\subsection{Data analysis}

The analysis of the data obtained with PAMELA during a period of about eight years has been described in detail in Ref. [1]. We illustrate schematically the main steps and then, in this paper, we deepen and extend the study of the data, in order to be more confident that some part of the cosmic rays measured by PAMELA comes from Jupiter.

The data have been provided by the PAMELA team [1] in three independent files according to the proton energy: protons with energy in the range $0.4-0.65 \mathrm{GeV}$, in the range $0.65-15 \mathrm{GeV}$ and in the range $15-50 \mathrm{GeV}$.

For each day, daily averages were calculated, from the beginning of the PAMELA operation, 9 July 2006-31 August 2014 with a few gaps in the data taking.

To ensure a clean galactic sample, contamination of solar particles from solar flares [33] has been avoided, discarding data taken during major solar particle events. Other shortterm effects on GCRs, like Forbush decreases, caused by coronal mass ejections passing through Earth, have been removed, even if their impact on the proton intensities appears negligible for most events. Information about the 


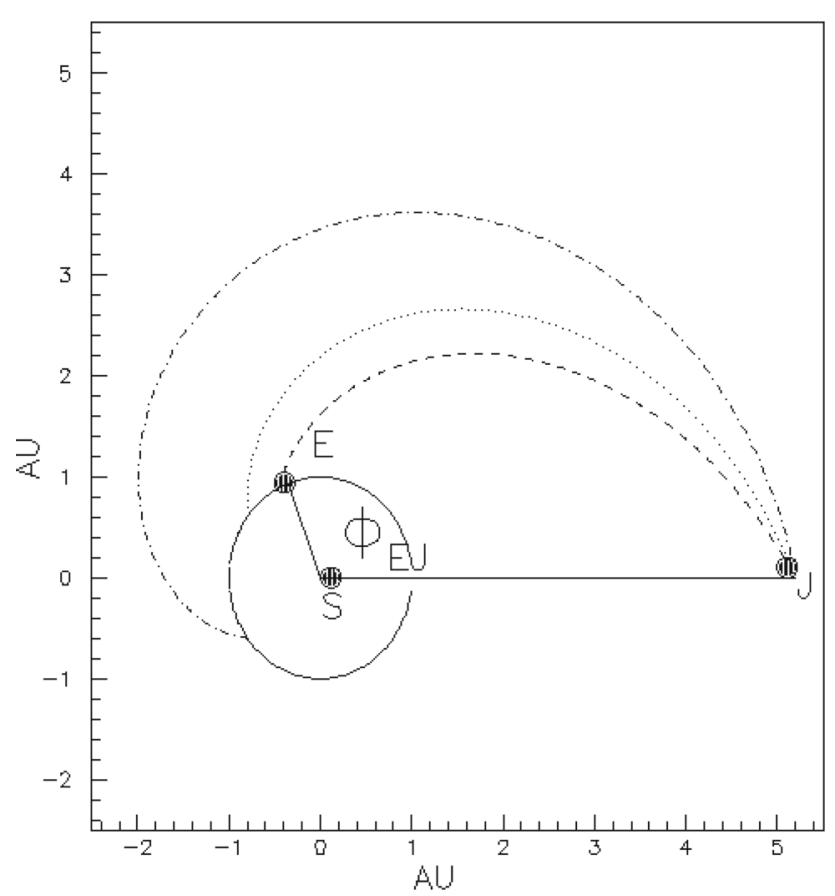

Fig. 1 A schematic view of Sun, Earth and Jupiter with the IMF lines connecting Jupiter with Earth, for $V_{R}=400 \mathrm{~km} / \mathrm{s}$. The dashed line refers to $\gamma=0.57$, the dotted line to $\gamma=0.37$. The dot-dashed line refers to the Parker model. (We notice that the IMF lines based on measurements intersect the Earth orbit at angles $\Phi_{E J}<180^{\circ}$ )

procedure adopted for obtaining the daily averages used in the present analysis can be found in [34,35].

A total of 2193 daily averages of proton fluxes have been obtained. At low energy the proton flux measured by PAMELA increased up to the year 2009 at sunspot minimum, then decreased when the solar activity increased.

To get rid of the long term variations associated with the solar cycle, the proton fluxes $f(t)$ have been fitted versus time and the fluctuations $\xi$ were evaluated.

$\xi(t)=f(t)-f i t$.

The data were fitted in each file with two distinct third degree $^{3}$ polynomials (one for the data before the maximum in the proton intensity, and one for data after the maximum), in the form $\xi(t)=a+b t+c t^{2}+d t^{3}$ excluding the time during the maximum ( $t$ indicates number of days from 9 July 2006, for both fits). The results (already shown in Fig. 1 of [1]) are given in Fig. 2.

We now study the behavior of $\xi(t)$ versus time, while PAMELA, orbiting around the Earth, makes about eight turns around the sun and we associate to each value $\xi(t)$ the angle $\Phi_{E J}(t)$. If our hypothesis is correct, when the Earth, during

\footnotetext{
${ }^{3}$ The smallest degree for a reasonable fit.
}
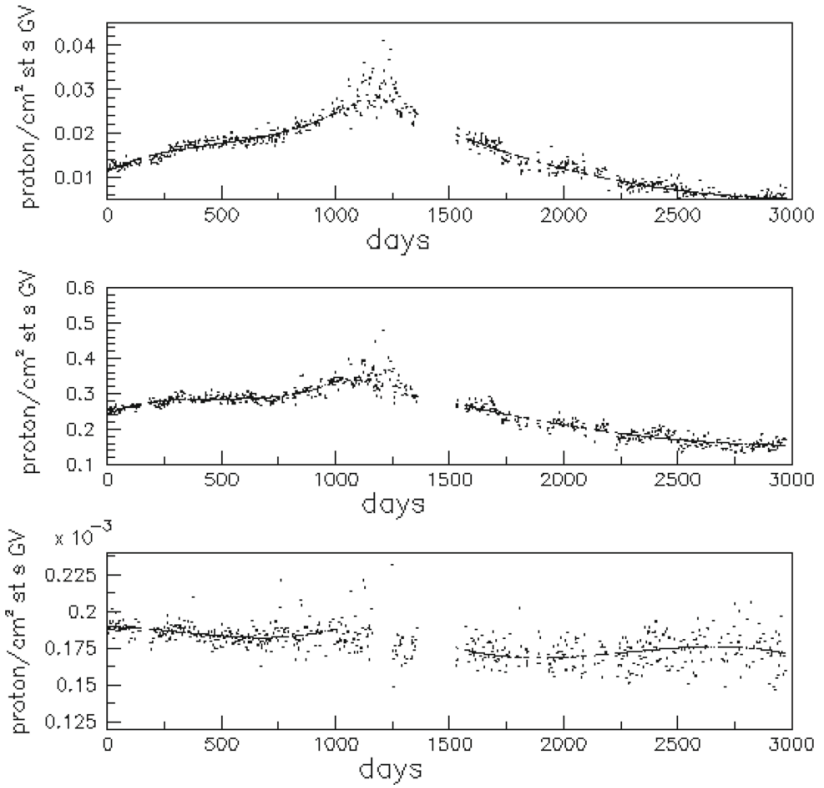

Fig. 2 Days from 9 July 2006. On upper graphs the daily proton flux (number of protons/ $\left(\mathrm{cm}^{2} \mathrm{sr} \mathrm{s} \mathrm{GV}\right)$ ) in the range $0.4-0.65 \mathrm{GV}$ versus time (1865 fitted daily averages). In the middle one protons in the range $0.65-15 \mathrm{GeV}$ (1866 fitted daily averages). In the lower one protons in the range $15-50 \mathrm{GeV}$ (1809 fitted daily averages)

the orbits around the Sun, intersects the lines of the IMF which come from Jupiter the proton flux should be enhanced.

In our previous study, looking at Fig. 1, we had noticed that the Earth intersects the lines of the measured IMF coming from Jupiter at angles $\Phi_{E J}<180^{\circ}$ and we had distributed the daily proton fluxes in two categories; (a) those with angles $\Phi_{E J} \leq 180^{\circ}$, (b) those with angles $\Phi_{E J}>180^{\circ}$.

Taking the averages of these two categories we had found [1] that the average proton flux for $\Phi_{E J}<180^{\circ}$ exceeded the average flux for $\Phi_{E J}>180^{\circ}$ by several standard deviations, namely about 12 standard deviations for rigidities in the range $0.4-0.65 \mathrm{GV}$, about 10 for rigidities in the range $0.65-15 \mathrm{GV}$ and 4 for rigidities in the range $15-50 \mathrm{GV}$.

Here we have decided to look for all possible directions, in order not to be biased by previous choices. For a given angle $\Phi$ we divide the daily averages in a series of two categories: (a) $N_{a}$ daily averages with $\Phi-90^{\circ}<\Phi_{E J}<\Phi+90^{\circ}$ and b) $N_{b}$ remaining daily averages, with $\Phi$ varying from $0^{\circ}$ to $180^{\circ}$ in steps of $1^{\circ}$ (the $\pm 90^{\circ}$ for taking care of large fluctuations due to the solar activity and to the solar wind variations). The case considered in [1], shown above, corresponds to the case $\Phi=90^{\circ}$.

For each category, which includes $N_{a, b}$ daily averages, the average value $\eta_{a}$ for category (a), the average value $\eta_{b}$ for category (b) and the standard deviations $\sigma_{a}$ and $\sigma_{b}$ of these two averages are calculated. We then calculate the quantities 
Table 2 Results. The $\Phi$ value at the center of the flat tops in Fig. 3 , the difference D between the average flux with $\Phi-90^{\circ}<\Phi_{E J}<$ $\Phi+90^{\circ}$ and the average flux outside this range, SNR and the percentage excess of cosmic ray calculated as D/(average flux). The average flux is obtained by the Fig. 2

\begin{tabular}{lllrl}
\hline $\begin{array}{l}\text { Rigidity } \\
\text { GV }\end{array}$ & $\begin{array}{l}\Phi \\
\pm 90^{\circ}\end{array}$ & $\begin{array}{l}\text { D } \\
N /\left(\mathrm{cm}^{2} \text { sr s GV }\right)\end{array}$ & SNR & $\begin{array}{l}\text { CR excess } \\
\sim \%\end{array}$ \\
\hline $0.4-0.65$ & 105 & $(6.50 \pm 0.52) 10^{-4}$ & 12.6 & 4.8 \\
$0.65-15$ & 100 & $(6.32 \pm 0.60) 10^{-3}$ & 10.5 & 2.7 \\
$15-50$ & 60 & $(1.82 \pm 0.41) 10^{-6}$ & 4.5 & 1
\end{tabular}
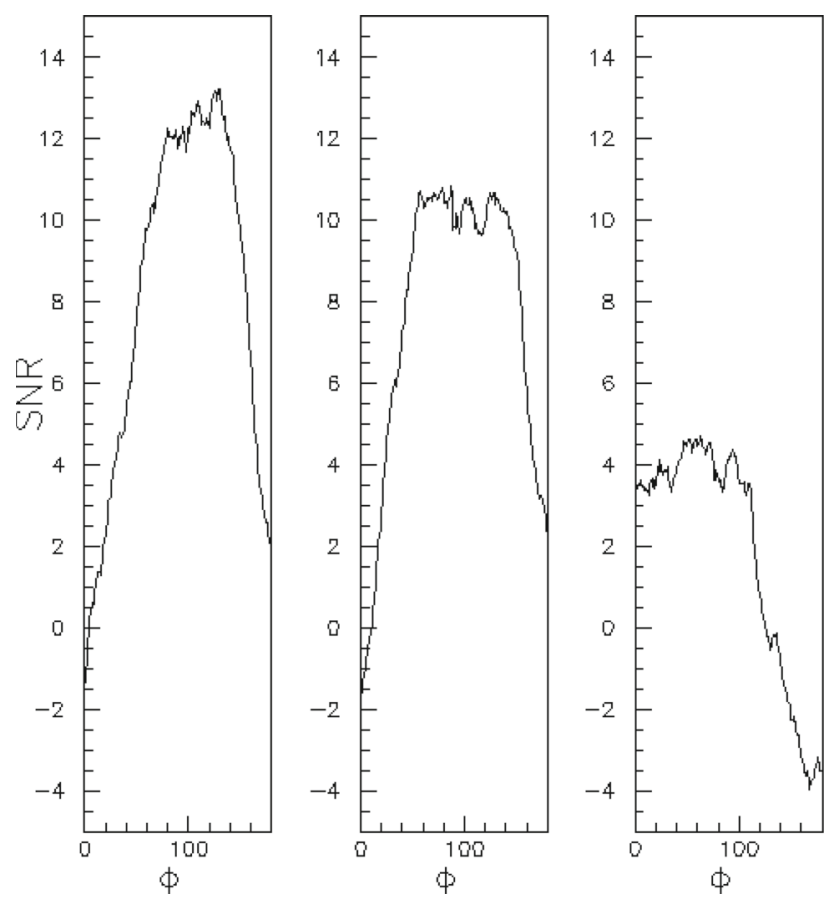

Fig. 3 The $S N R=D / \sigma_{\text {total }}$ versus $\Phi$. Left graph for the rigidities $0.4-0.65 \mathrm{GV}$, middle graph for $0.65-15 \mathrm{GV}$, right graph for $15-50 \mathrm{GV}$

$$
\begin{aligned}
D & =\eta_{a}-\eta_{b} \\
\sigma_{\text {total }} & =\sqrt{\sigma_{a}^{2}+\sigma_{b}^{2}} .
\end{aligned}
$$

If the proton fluxes are distributed uniformly along the Earth orbit we expect $\mathrm{D}=0$, within errors.

The result is shown in the Table 2 and in Fig. 3 where $S N R=D / \sigma_{\text {total }}$ (number of standard deviations) is given versus $\Phi$.

If we want to associate the SNR to the probability that the result was due to chance, we must verify that the data $\xi(t)$ have gaussian distribution. This is done in Fig. 4 for the rigidity $0.4-0.65 \mathrm{GV}$ and the case $129^{\circ}-90^{\circ}<\Phi_{E J}<$ $129^{\circ}+90^{\circ}$, when SNR $=13.2$ is the largest one.

The upper graph gives the distribution of all the daily averages $\left(0^{\circ}<\Phi_{E J}<360^{\circ}\right)$. The distribution is gaussian and the average $\eta_{\text {total }}=(-0.26 \pm 0.26) 10^{-4}$ is null, as expected. We also find that the distributions for the two
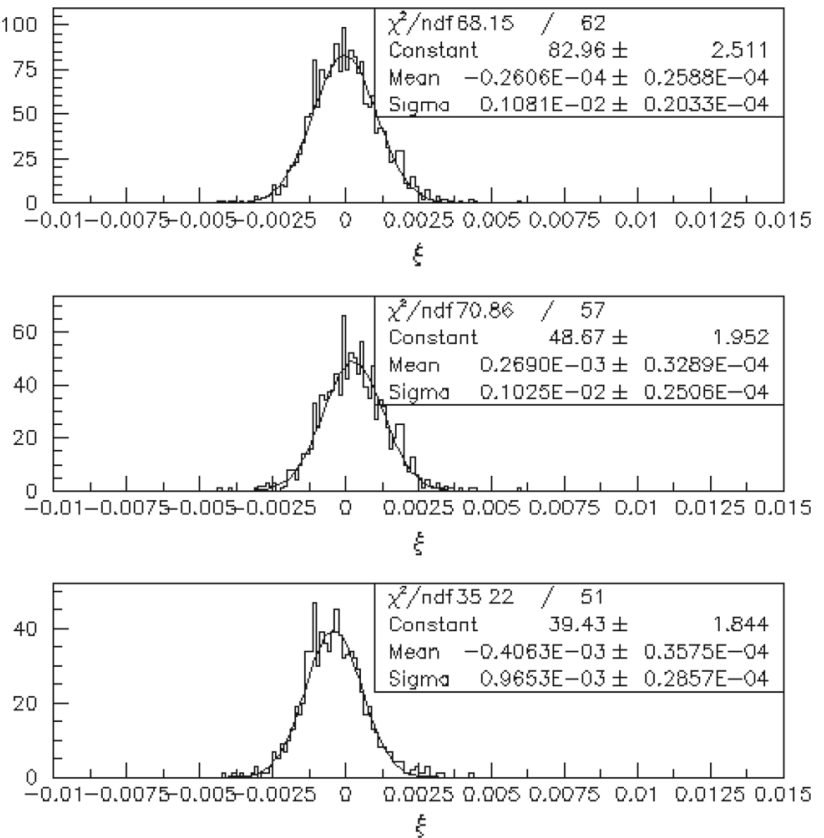

Fig. 4 Distribution of $\xi(t)$ for rigidity $0.4-0.65 \mathrm{GV}$. On the top the gaussian distribution for all $\xi(t)$ with 1865 daily averages $\left(0^{\circ}<\Phi_{E J}<\right.$ $360^{\circ}$ ), in the middle the distribution with 1069 daily averages having $129^{\circ}-90^{\circ}<\Phi_{E J}<129^{\circ}+90^{\circ}$, on the bottom the distribution with 796 daily averages having $\Phi_{E J}$ outside the above angle interval

categories a) and b) (middle and lower graph) are both gaussian and have different averages, $\eta_{a}=(2.69 \pm 0.33) 10^{-4}$ and $\eta_{b}=(-4.06 \pm 0.36) 10^{-4}$.

Using these two averages of the two gaussian distributions we get $D=\eta_{a}-\eta_{b}=(6.75 \pm 0.49) 10^{-4}$, that is $D \neq 0$ with $S N R=\frac{0.680}{0.049}=13.9$ standard deviations. Using the averages of the measured daily data we get $\mathrm{SNR}=13.2$, as shown in Fig. 3.

We conclude that the asymmetric behavior of the proton fluxes is a real phenomenon, not due to chance.

\section{Discussion}

The results shown in Fig. 3 give important information on the behavior of the proton fluxes measured by PAMELA.

Starting from the graph on the left, for rigidities $0.4-0.65$ $\mathrm{GV}$, we notice that SNR is roughly flat from $\Phi=80^{\circ}$ to $\Phi=130^{\circ}$ at values SNR $=12-13$, reaching the maximum $\mathrm{SNR}=13.2$ at $\Phi=129^{\circ}$.

We interpret this as follows:

consider the $\Phi=105^{\circ}$ value at the center of the flat top of Fig. 3 where SNR $=12.6$ (see the Table 2). The large SNR indicates that the average proton flux when PAMELA, during its orbit around the Sun, is at locations where $105^{\circ}-90^{\circ}<$ $\Phi_{E J}<105^{\circ}+90^{\circ}$ is certainly greater than the average flux when $\Phi_{E J}$ is outside that range. 
From Table 1 and Fig. 1, we deduce that the average proton flux is higher when PAMELA intercepts the IMF lines coming from Jupiter. The top flatness is due to the fluctuations of the IMF and especially is due to the variations of the solar wind speed that cause the IMF lines to sweep the entire quadrant from $0^{\circ}$ to $180^{\circ}$.

The graph in the middle, for rigidities $0.65-15 \mathrm{GV}$, also shows a larger average proton flux from $\Phi=60^{\circ}$ to $\Phi=$ $140^{\circ}$, with $S N R \simeq 10$, smaller than that at lower rigidity.

The graph on the right, for rigidities $15-50 \mathrm{GV}$, also has a flat top, from $\Phi=20^{\circ}$ to $\Phi=100^{\circ}$ and $S N R \simeq 4$.

A possible, plausible explanation for these differences is that, while at low energy the proton Larmor radius is small, about $0.01 \mathrm{AU}$ for $0.65 \mathrm{GeV}$, meaning that the protons can follow closely the IMF lines, at higher energies the Larmor radius is greater, $0.3 \mathrm{AU}$ for a $50 \mathrm{GeV}$ proton, so the high energy protons might not follow closely, on average during the 8 years of measurements, the IMF lines and might have a straighter path that intersect Earth's orbit at smaller $\Phi_{E J}$.

The different values of SNR indicate that the contribution of the proton flux from Jupiter decreases with increasing energy. This is likely related to the cutoff energy of Jupiter which limits the production of high energy protons.

In conclusion we find a clear excess of proton fluxes at $\Phi_{E J}$ angles where the IMF lines of force from Jupiter, calculated from the experiments, intersect the Earth's orbit, for all proton energies. We have also verified (see [1]), that the enhancement of the proton fluxes at $\Phi_{E J}$ angles smaller than $180^{\circ}$ occurs separately for all energies both before and after day 1200 of Fig. 2.

\section{Conclusion}

The discovery of the Van Allen belt in a magnetosphere due to the dipole field of the Earth showed, already in 1958, that electrically charged particle are concentrated in certain regions of space where acceleration mechanisms operate. Thus the idea that the magnetospheres leak out the most energetic particles comes out in a natural way.

The vicinity of a powerful magnetosphere, as that possessed by the planet Jupiter, has allowed to test if part of the cosmic rays observed on Earth comes from Jupiter. The test has given a positive result with over ten standard deviations, that happens very rarely when new experimental data are studied.

If another cause is proposed to explain the observed effect, it must account for why, on average over a period of 8 years, the high energy interplanetary proton fluxes are larger, beyond any reasonable doubt, when the Earth is at a particular position in its orbit around the Sun.

If the most simple explanation of a Jovian contribution is valid, the theory that pulsars be one of the candidates of the observed ultra-high-energy cosmic rays is reinforced and makes reasonable to extrapolate this feature to all astrophysical magnetospheres where powerful acceleration mechanisms operate (say: Pulsars, AGN, Black Holes...).

Acknowledgements I thank Piergiorgio Picozza and his reserch group for the PAMELA data. I am indebted with Guido Barbiellini and Olga Khabarova for suggestions and discussions and with Carl E. McIlwain and Marcello Piccolo for suggestions. I also thank two unknown referees for their important comments.

Open Access This article is distributed under the terms of the Creative Commons Attribution 4.0 International License (http://creativecomm ons.org/licenses/by/4.0/), which permits unrestricted use, distribution, and reproduction in any medium, provided you give appropriate credit to the original author(s) and the source, provide a link to the Creative Commons license, and indicate if changes were made. Funded by SCOAP ${ }^{3}$.

\section{References}

1. O. Adriani et al., Unexpected cyclic behavior in cosmic-ray protons observed by PAMELA at 1au. Astrophys. J. Lett. 85, L28 (2018)

2. O. Adriani, The PAMELA mission: heralding a new era in precision cosmic ray physics. Phys. Reports 544, 323 (2014)

3. G. Pizzella, Emission of cosmic rays by a dipole magnetosphere. Nature 226, 434 (1970)

4. J.A. Van Allen, Magnetospheres, Cosmic Rays, and the Interplanetary Medium, The New Solar System, 3rd edn. (Sky Publishing Corporation and Cambridge University Press, Cambridge, 1990)

5. T. Saito, Space Sci. Rev. 10, 319 (1969). (see for instance)

6. D.A. Gurnett et al., J. Geophys. Res. 74, 4631 (1969)

7. C.T. Russell, R.E. Holzer, E.J. Smith, J. Geophys. Res. 75, 755 (1970)

8. A.J. Dessler, V.E. Francis, E.N. Parker, Geomagnetic storm sudden commencement rise times. J. Geophys. Res. 65, 2715 (1960)

9. G.D. Reeves, Electron acceleration in the heart of the van allen radiation belts. Science 341(6149), 991-994 (2013)

10. Jovian radiation belts. https://www.spenvis.oma.be/help/ background/planetary/traprad_jup.html. Accessed 2018

11. J.A. Simpson, Protons and electrons in jupiter's magnetic field: results from the University of Chicago experiment on Pioneer 10. Science 183(4122), 306-309 (1974)

12. B. H. Mauk et al., Energetic ion characteristics and neutral gas interactions in Jupiter's magnetosphere J. Geophys. Res, 109, A09S12 (2004). https://doi.org/10.1029/2003JA010270

13. B. Heber, M.S. Potgieter, Cosmic rays at high heliolatitudes. Space Sci. Rev. 127(14), 117194 (2006)

14. T. Gold, Rotating neutron stars and the nature of the pulsars. Nature 221, 25 (1969)

15. E. Fermi, On the origin of the cosmic radiation. Phys. Rev. 75, 1169 (1949)

16. The Pierre Auger Collaboration, Correlation of the HighestEnergy Cosmic Rays with Nearby Extragalactic Objects. Science 318(5852), 938 (2007)

17. A.M. Hillas, The origin of ultra high-energy cosmic rays. Ann. Rev. Astrom. Astrophys. 22, 425-444 (1984)

18. H. Alfven, C.G. Falthammar, Cosmic electrodynamics (Oxford at the Clarendom Press, Oxford, 1963)

19. J. Carlsson, Breakdown of adiabatic invariance in spherical tokamaks. Phys. Plasmas 8, 4725 (2001). arXiv:physics/0105105 
20. G. Pizzella, G. Venditti: Evidence for emission of cosmic rays by Jupiter. In: Proceedings of the XIII international conference on cosmic rays, Vol. 2 (1973), p. 1129

21. B. Mitra et al., Cosmic rays from jupiter. Lett. Nuovo Cimento 33, N19 (1982)

22. B. Mitra et al., Probable Contribution to Cosmic Rays by Jupiter. In: Proc. 18th ICRC, Volume 3, p. 182, Bangalore (1983)

23. D.B. Swinson, The possible influence of jupiter on cosmic radiation at the earth. JGR 79, 3695 (1974)

24. K. Nagashime, R. Tatsuoka, No sign of cosmic-ray generation from jupiter, detectable at ground-based stations. Il Nuovo Cimento 7C, 379 (1984)

25. E.N. Parker, Dynamic of interplanetary gas and magnetic field. Astrophys. J. 128, 664 (1958)

26. E.J. Smith, A. Balogh, M.E. Burton, R. Forsyth, R.P. Lepping, Radial and Azimuthal components of the heliospheric magnetic field: Ulysses observations. Adv. Space Res. 20(1), 47-53 (1997)

27. O.V. Khabarova, The interplanetary magnetic field: radial and latitudinal dependences. Astron. Rep. 57(11), 844859 (2013)

28. K.W. Behannon, Heliocentric distance dependence of the interplanetary magnetic field. Rev. Geophys. 16, 125-145 (1978)
29. C.W. Smith, J.W. Bieber, The interplanetary magnetic field. Astrophys. J. 370, 435-441 (1991)

30. D. Winterhalter, E.J. Smith, J.H. Wolfe, J.A. Slavin, Spatial gradients in the heliospheric magnetic field: Pioneer 11 observations between $1 \mathrm{AU}$ and $24 \mathrm{AU}$, and over solar cycle 21. J. Geophys. Res. 95, 1-11 (1990)

31. E.J. Smith, A. Balogh, M.E. Burton, R. Forsyth, R.P. Lepping, Radial and azimuthal components of the heliospheric magnetic field: Ulysses observations. Adv. Space Res. 20, 47-53 (1997)

32. O. Khabarova, V. Obridko, Puzzles of the interplanetary magnetic field in the inner heliosphere. Astrophys. J. 761, 82 (2012)

33. A. Bruno et al., Solar energetic particle events observed by the PAMELA mission. Astrophys. J. 862(2), 97 (2018)

34. O. Adriani et al., Time dependence of the proton flux measured by PAMELA during the July 2006-December 2009 solar maximum. (2013). arXiv:1301.4108v2 [astro-ph.HE]

35. M. Martucci et al., Proton fluxes measured by the PAMELA experiment from the maximum to the maximum solar activity for the 24th solar cycle. https://arxiv.org/pdf/1801.07112.pdf (2018) 